\title{
Xanthine in urine stimulates growth of Leishmania promastigotes in vitro
}

The leishmaniases are parasitic diseases that affect large populations in vast areas of the world (Desjeux, 2001). The causative agents of these diseases, protozoan parasites belonging to the genus Leishmania (Kinetoplastida:

Trypanosomatidae), are transmitted by phlebotomine sand flies (Killick-Kendrick, 1999). In culture media (at $26-28{ }^{\circ} \mathrm{C}, \mathrm{pH}$ 7.2), Leishmania parasites develop as motile promastigotes similar to those found in the sand fly midgut. A number of reports have shown that the addition of $1-$ $5 \%$ human urine stimulates growth, leading to more rapid multiplication and a higher concentration of parasites at the stationary phase (Ali et al., 1998;

Armstrong \& Patterson, 1994; Howard et al., 1991; Iqbal et al., 2006; Shamsuzzaman et al., 1999; Singh et al., 2000). Preliminary studies have indicated that the factor responsible for this enhancement is a small molecule which is not destroyed by autoclaving (Ali et al., 1998). However, despite the substantial advantages of using defined media for the culture of human pathogens (Schuster \& Sullivan, 2002), the factor(s) in urine that is responsible for promoting growth of Leishmania has not been identified.

Preliminary experiments confirmed that the addition of $5 \%(\mathrm{v} / \mathrm{v})$ human urine to Leishmania major promastigotes cultured in RPMI 1640 enhanced the rate of multiplication about 10-fold (two-tailed $t$-test, $P<0.005)$. The final concentration at stationary phase increased from $4.0 \times 10^{6}$ to almost $4.2 \times 10^{7}$ parasites $\mathrm{ml}^{-1}$. This exacerbative effect was not abolished by boiling the urine or treating it with proteinase K (Fig. 1).

Since Leishmania parasites are auxotrophic for purines and human urine contains low concentrations of these molecules (Boitz \& Ullman, 2006; Bromberg et al., 1957a, b), we tested whether adenine, hypoxanthine and xanthine $(10 \mu \mathrm{M})$ enhanced the growth of L. major. Of these, only xanthine

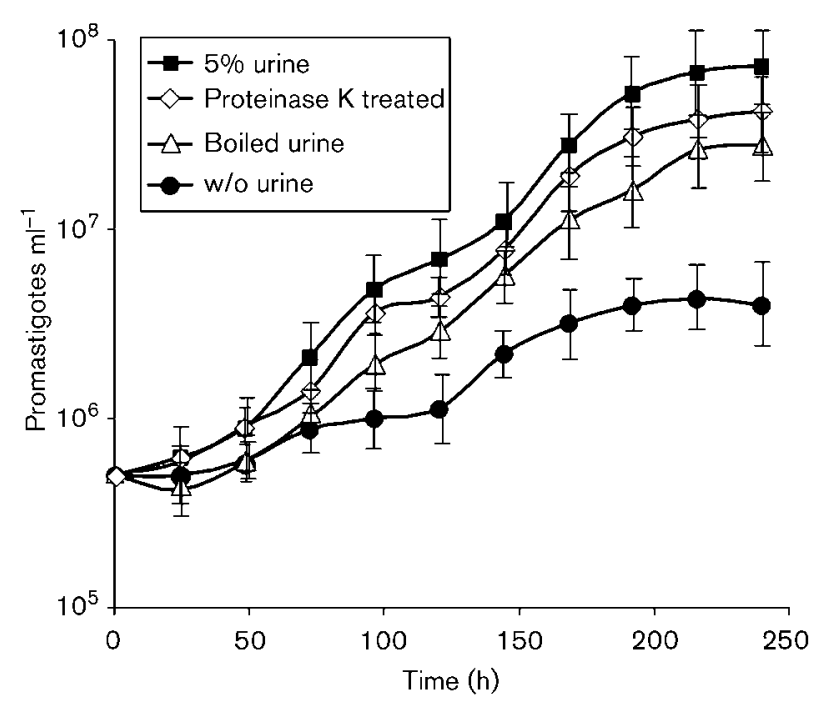

Fig. 1. Growth curves of Leishmania major in RPMI 1640. Addition of $5 \%$ human urine to the medium significantly enhances growth rate and prolongs the stationary phase. Enhancement is not abrogated by boiling or proteinase $\mathrm{K}$ treatment. Experiments were repeated three times.

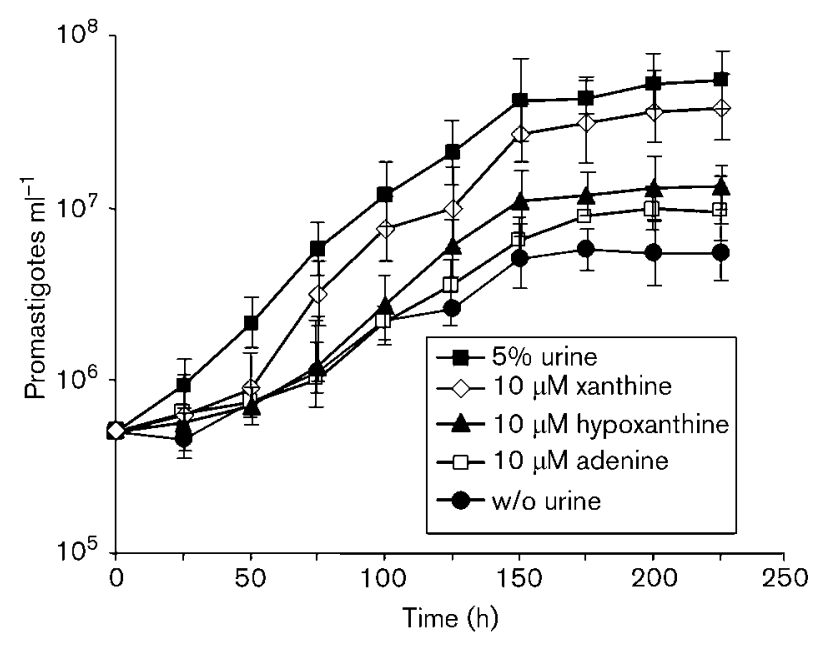

Fig. 2. Growth curves of Leishmania major in RPMI 1640 with added purines $(10 \mu \mathrm{M})$. Xanthine enhances growth almost as much as $5 \%$ human urine. The experiment was repeated four times. 

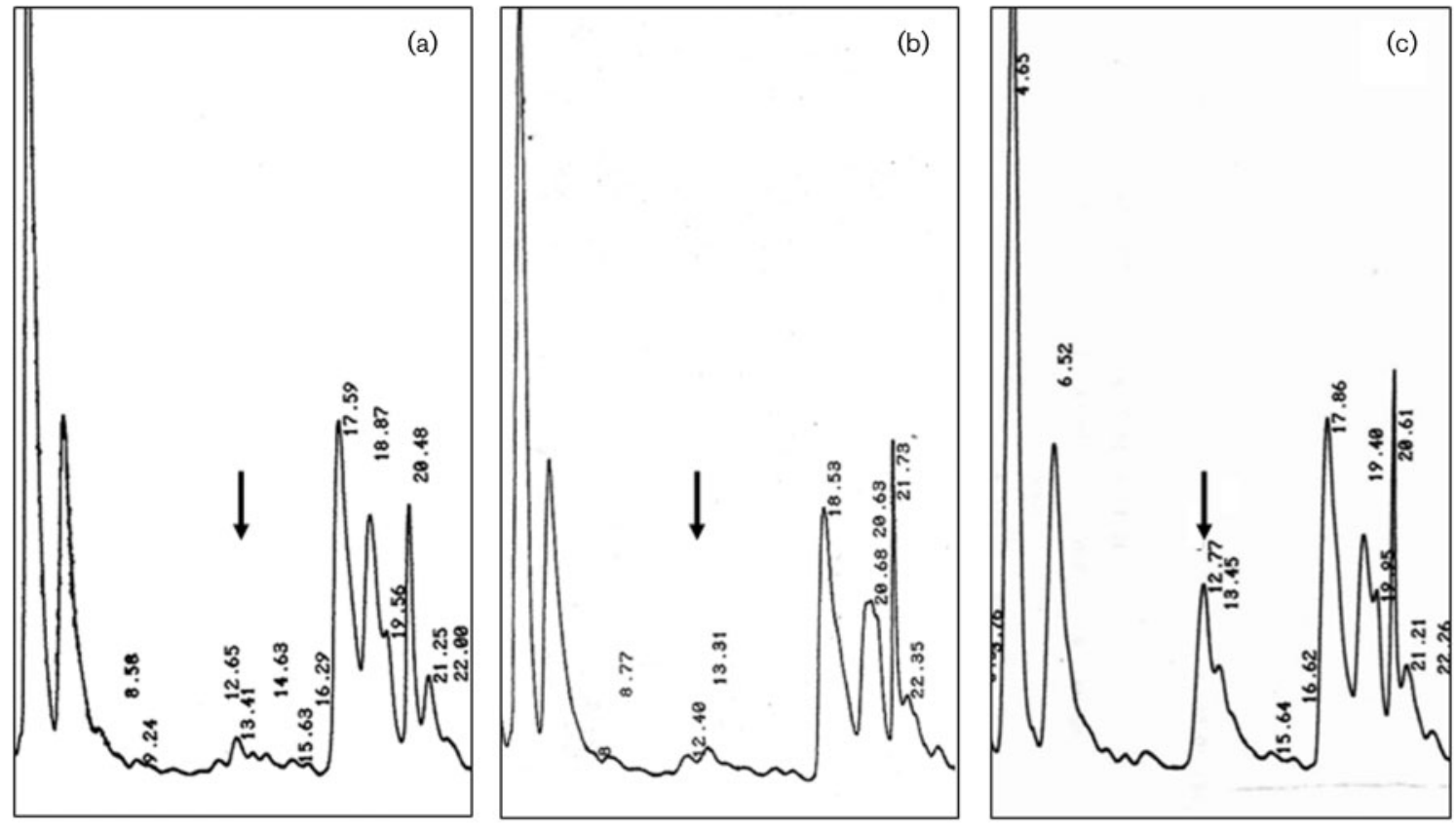

Fig. 3. HPLC analysis of human urine. (a) Chromatogram of normal urine. (b) Chromatogram of urine depleted of xanthine after passing through an oligonucleotide purification cartridge. (c) Chromatogram of xanthine-depleted urine to which $10 \mathrm{mM}$ synthetic xanthine was added. Arrows point to peaks corresponding to xanthine.

$(10 \mu \mathrm{M})$ significantly and repeatedly enhanced the growth of promastigotes in vitro (two-tailed $t$-test, $P<0.005$ ) (Fig. 2 ).

In order to ascertain whether xanthine is the major component in urine that enhances leishmanial growth in vitro, fresh urine was depleted of xanthine by passing it through an activated oligonucleotide purification cartridge (OPC) (Applied Biosystems) according to a variation of the manufacturer's protocol as described previously (Deutsch et al., 1997). Depletion was demonstrated by HPLC analysis (Fig. 3). The peak at RT $13.41 \mathrm{~min}$ corresponding to xanthine (Fig. 3a) was markedly smaller after passing the urine through an OPC (Fig. 3b; RT 13.31). The observed deviations in RT were minor and reasonable for inter-days deviations (measuring samples on different days). Addition of exogenous xanthine restored a peak at almost the same RT (Fig. 3c, with minor changes due to the different extent of ionization of the synthetic xanthine).

Finally, to make sure that xanthine is the main component that enhances multiplication of Leishmania in vitro, the three urine types shown in Fig. 3 were used to supplement RPMI 1640 culture medium at $5 \%(\mathrm{v} / \mathrm{v})$. Results depicted in Fig. 4 confirm that depletion of xanthine from human urine abrogates its capacity to enhance growth of $L$. major promastigotes (two-tailed $t$-test, $P<0.005)$. Moreover, addition of $10 \mu \mathrm{M}$ xanthine to the urine restored this capacity (Fig. 4 ).
Unlike mammalian cells that synthesize purines de novo, all protozoan parasites studied thus far are incapable of synthesizing the purine ring, rendering them auxotrophic for purines (Marr et al., 1978). Xanthine is excreted in human

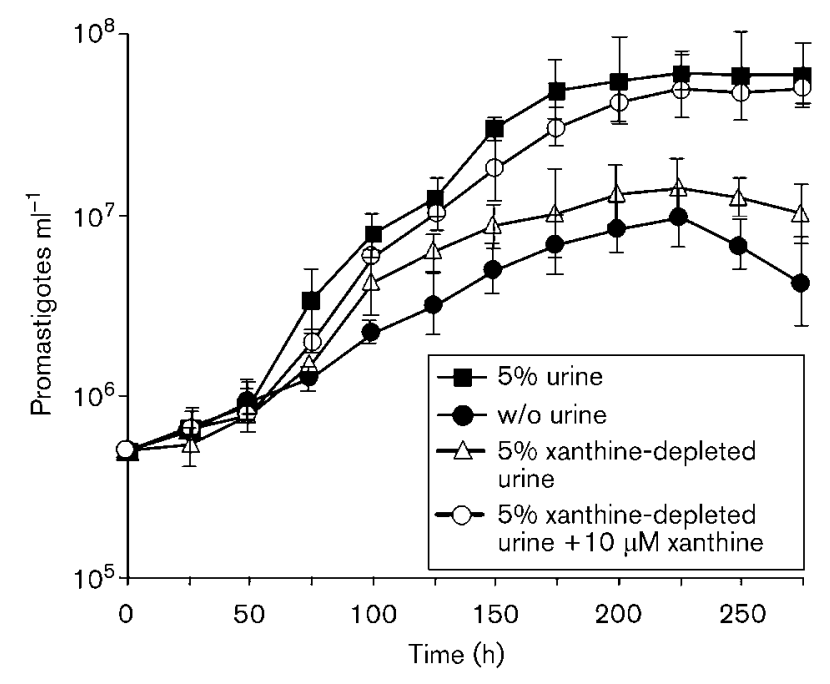

Fig. 4. Growth curves of Leishmania major in RPMI 1640 supplemented with $5 \%$ normal urine, xanthine-depleted urine or xanthine-depleted urine supplemented with $10 \mu \mathrm{M}$ xanthine (final concentration). 
urine at approximately $10 \mathrm{mg}$ per $24 \mathrm{~h}$, and its concentration in normal urine is around $0.2-0.5 \mathrm{mM}$. Our data show that like $5 \%(\mathrm{v} / \mathrm{v})$ human urine, $10 \mu \mathrm{M}$ xanthine significantly enhances the growth of $L$. major promastigotes in culture (Fig. 2). Furthermore, the depletion of xanthine from urine abrogated its capacity to exacerbate growth of Leishmania promastigotes (Fig. 4). Although passage of the urine through the OPC may have removed other purines as well, we showed that the exacerbative effect can be restored almost entirely by adding commercial xanthine at $10 \mu \mathrm{M}$ (Fig. 4). The results strongly suggest that xanthine is the one most active molecule in human urine that enhances the growth of L. major promastigotes in culture, and its routine addition at $10 \mu \mathrm{M}$ should improve the culture of Leishmania in vitro (Merlen et al., 1999; Schuster \& Sullivan, 2002).

\section{Acknowledgements}

This research was supported by grant number 235/99-2 from 'The Israel Science Foundation' founded by the Israeli Academy of Sciences and Humanities.

\section{Alon Warburg, ${ }^{1}$ Shoshana Gelman ${ }^{1}$ and Joseph Deutsch ${ }^{2}$}

${ }^{1}$ Department of Parasitology, The Kuvin Centre for the Study of Infectious and
Tropical Diseases, The Hadassah Medical School, Jerusalem 91120, Israel

${ }^{2}$ School of Pharmacy, The Faculty of Medicine, The Hebrew University of Jerusalem, Jerusalem 91120, Israel

Correspondence: Alon Warburg (warburg@cc.huji.ac.il)

Ali, S. A., Iqbal, J., Ahmad, B. \& Masoom, M. (1998). A semisynthetic fetal calf serum-free liquid medium for in vitro cultivation of Leishmania promastigotes. Am J Trop Med Hyg 59, 163-165.

Armstrong, T. C. \& Patterson, J. L. (1994). Cultivation of Leishmania braziliensis in an economical serum-free medium containing human urine. J Parasitol 80, 1030-1032.

Boitz, J. M. \& Ullman, B. (2006). Leishmania donovani singly deficient in HGPRT, APRT or XPRT are viable in vitro and within mammalian macrophages. Mol Biochem Parasitol 148, 24-30.

Bromberg, P. A., Gutman, A. B. \& Weissmann, B. (1957a). The purine bases of human urine. I. Separation and identification. J Biol Chem 224, 407-422.

Bromberg, P. A., Gutman, A. B. \& Weissmann, B. (1957b). The purine bases of human urine. II. Semiquantitative estimation and isotope incorporation. J Biol Chem 224, 423-434.

Desjeux, P. (2001). Worldwide increasing risk factors for leishmaniasis. Med Microbiol Immunol 190, 77-79.

Deutsch, J., Rapoport, S. I. \& Purdon, A. D. (1997). Relation between free fatty acid and acyl-CoA concentrations in rat brain following decapitation. Neurochem Res 22, 759-765.
Howard, M. K., Pharoah, M. M., Ashall, F. \& Miles, M. A. (1991). Human urine stimulates growth of Leishmania in vitro. Trans $R$ Soc Trop Med Hyg 85, 477-479.

Iqbal, J., Jamshid, M., Ahmed, B., Bukhari, I., Bashir, S. \& Yasinzai, M. M. (2006). Some studies on human urine as promoter for the growth of leishmania in vitro. Pak J Pharm Sci 19, 152-155.

Killick-Kendrick, R. (1999). The biology and control of phlebotomine sand flies. Clin Dermatol 17, 279-289.

Marr, J. J., Berens, R. L. \& Nelson, D. J. (1978). Purine metabolism in Leishmania donovani and Leishmania braziliensis. Biochim Biophys Acta 544, 360-371.

Merlen, T., Sereno, D., Brajon, N., Rostand, F. \& Lemesre, J. L. (1999). Leishmania spp: completely defined medium without serum and macromolecules (CDM/LP) for the continuous in vitro cultivation of infective promastigote forms. Am J Trop Med Hyg 60, 41-50.

Schuster, F. L. \& Sullivan, J. J. (2002). Cultivation of clinically significant hemoflagellates. Clin Microbiol Rev 15, 374-389.

Shamsuzzaman, S. M., Furuya, M., Korenaga, M., Imamura, K. \& Hashiguchi, Y. (1999). Use of urine samples from healthy humans, nephritis patients or other animals as an alternative to foetal calf serum in the culture of Leishmania ( $L$.) donovani in vitro. Ann Trop Med Parasitol 93, 613-620.

Singh, S., Mohapatra, D. P. \& Sivakumar, R. (2000). Successful replacement of fetal calf serum with human urine for in vitro culture of Leishmania donovani. J Commun Dis 32, 289-294. 